\title{
Materials Informatics Approach to the Identification of One-Band Correlated Materials Analogous to the Cuprates
}

\author{
Eric B. Isaacs and Chris Wolverton* \\ Department of Materials Science and Engineering, Northwestern University, \\ Evanston, Illinois 60208, USA
}

\begin{abstract}
(Received 27 June 2018; revised manuscript received 22 January 2019; published 30 May 2019)
\end{abstract}
\begin{abstract}
One important yet exceedingly rare property of the cuprate high-temperature superconductors is the presence of a single correlated $d$ band in the low-energy spectrum, leading to the one-band Hubbard model as the minimal description. In order to search for materials with interesting strong correlation physics as well as possible benchmark systems for the one-band Hubbard model, here we present a new approach to find one-band correlated materials analogous to the cuprates by leveraging the emerging area of materials informatics. Using the composition, structure, and formation energy of more than half a million real and hypothetical inorganic crystalline materials in the Open Quantum Materials Database, we search for synthesizable materials whose nominal transition-metal $d$ electron count and crystal field are compatible with achieving an isolated half-filled $d$ band. Thirteen $\mathrm{Cu}$ compounds, including bromide, oxide, selenate, borate, pyrophosphate, hydrogen phosphate, and pyrosilicate chemistries, and one Fe oxide compound are shown to successfully achieve the one-band electronic structure based on density-functional theory band structure calculations. Further calculations including magnetism and explicit on-site Coulomb interaction, performed for a representative subset of five candidate materials, reveal significant evidence for strong correlation physics, including Mott insulating behavior and antiferromagnetism. The success of our datadriven approach to discovering new correlated materials opens up new avenues to design and discover materials with rare electronic properties.
\end{abstract}

DOI: 10.1103/PhysRevX.9.021042

Subject Areas: Materials Science,

Strongly Correlated Materials

\section{INTRODUCTION}

The cuprates, one of the most famous classes of materials in condensed matter physics, are layered copper oxide ceramics with copper-oxygen planes exhibiting unconventional, high-temperature superconductivity [1]. Despite decades of study, the details of the mechanism and phase diagram of this class of materials are still not fully settled, due in part to a complex phase diagram in which doping the antiferromagnetic Mott insulating parent compound can lead to pseudogap, charge-density wave, spin-density wave, and "strange metal" phases in addition to the superconducting phase. However, it is generally accepted that the presence of a single $d$ orbital at low energy is a very important characteristic leading to the high critical temperature $T_{c}$ for superconductivity in the cuprates $[2,3]$.

\footnotetext{
cc-wolverton@northwestern.edu
}

Published by the American Physical Society under the terms of the Creative Commons Attribution 4.0 International license. Further distribution of this work must maintain attribution to the author(s) and the published article's title, journal citation, and DOI.
A minimal model for the physics of the cuprates is the one-band Hubbard model (1BHM) corresponding to the Hamiltonian

$$
\hat{H}_{1 \mathrm{BHM}}=-t \sum_{i, j, \sigma} \hat{c}_{i \sigma}^{\dagger} \hat{c}_{j \sigma}+U \sum_{i} \hat{n}_{i \uparrow} \hat{n}_{i \downarrow}
$$

Here, $\hat{c}_{i \sigma}^{(\dagger)}$ annihilates (creates) an electron of spin projection $\sigma$ on lattice site $i, \hat{n}_{i \sigma}=\hat{c}_{i \sigma}^{\dagger} \hat{c}_{i \sigma}, t$ is the hopping parameter, and the on-site Coulomb repulsion $U$ parametrizes the strength of the electronic correlations. Although the 1BHM is easy to write down, this manybody Hamiltonian has been solved in only one (using the Bethe ansatz [4]) and infinite (using dynamical mean-field theory [5]) dimensions. In general, $t$ leads to delocalized electronic states and metallic behavior, whereas $U$ localizes the electrons. In correlated materials like the parent compounds of the cuprates, $U$ is large with respect to $t$ and (Mott) insulating behavior is found.

Thus, the cuprates can be considered correlated oneband materials. We are not aware of any correlated oneband material outside of the cuprates, though $\mathrm{Sr}_{2} \mathrm{IrO}_{4}$ (isostructural to the cuprate parent compound $\mathrm{La}_{2} \mathrm{CuO}_{4}$ ) is a much-studied candidate due to the single $j=1 / 2$ band 
related to strong spin-orbit coupling [6]. One key reason for the rarity of one-band correlated materials is that most transition-metal (TM) compounds have octahedral or tetrahedral coordination, for which the five $d$ orbitals split into a group of three ( $T_{2 g}$ for octahedral) and a group of two ( $E_{g}$ for octahedral) according to group theory. Therefore, while a multiband Hubbard model is considered to be achieved in many compounds, realizations of the 1BHM are very rare. The discovery of such materials would be highly desirable for two purposes:

(1) to search for new unconventional superconductors or materials exhibiting other interesting strong correlation phenomena, and

(2) to provide physical realizations of the $1 \mathrm{BHM}$ to serve as benchmarks for our theories of strong correlation physics.

Recent efforts to achieve such a material have not been successful. For example, Chaloupka and Khaliullin proposed to break the symmetry of the $E_{g}$ levels in octahedral $\mathrm{LaNiO}_{3}$ by forming a superlattice with $\mathrm{LaAlO}_{3}$ [7]. Unfortunately, the experiments suggest that this approach yields insufficient symmetry breaking to achieve a oneband model [8]. $\mathrm{LiCuF}_{3}$ and related compounds were proposed in the trigonal bipyramidal coordination, whose symmetry does lead to a singly degenerate level (and which we thus call a "low-degeneracy" coordination) [9]. Although it was shown to have promising characteristics, $\mathrm{LiCuF}_{3}$ is not thermodynamically stable under ambient conditions [9].

Here, we employ the emerging area of materials informatics [10-12] to accelerate the search for one-band correlated materials analogous to the cuprates. We aim to answer the question, Can we identify correlated electron materials described by the one-band Hubbard model from an existing database of band theory calculations and, if so, are they likely to host Mott insulator, antiferromagnetism, or other strong correlation physics behavior? In order to identify candidate materials, we devise a materials database search strategy based on (1) the elements contained in the material, (2) the local coordination geometry of atoms in the crystal structure, (3) nominal valence electron count for each element, and (4) thermodynamic stability. In particular, we look for (1) compounds containing transition metals and anions, (2) transition metals coordinated by anions in a coordination environment whose crystal field leads to a singly degenerate $d$ level, (3) transition metals with a nominal $d$ electron count leading to half filling of such a level, and (4) compounds that are thermodynamically stable or nearly stable. For example, the cuprate parent compound $\mathrm{La}_{2} \mathrm{CuO}_{4}$ would satisfy criteria (2) and (3) since $\mathrm{Cu}$ is in a square planar environment and nominally in a $d^{9}$ configuration, half filling a singly degenerate $B_{1 g}\left(d_{x^{2}-y^{2}}\right)$ level. We query for any crystal that simultaneously satisfies all four criteria. We execute our strategy using the Open Quantum Materials Database (OQMD), an extensive electronic structure database with calculations for over 550000 real and hypothetical inorganic crystalline materials (as of June 2017). While previous attempts to identify candidate materials typically rely on expert intuition and focus on a small number of (often experimentally known) candidate materials [7,9,13-21], our systematic, data-driven approach allows for the identification of unstudied and even hypothetical candidate materials in a high-throughput manner.

There are two major findings in this study. The first is the successful identification of 14 one-band correlated materials: $\mathrm{Li}_{2} \mathrm{CuO}_{2}, \mathrm{CuBr}_{2}, \quad(\mathrm{Sr} / \mathrm{Ba}) \mathrm{Cu}\left(\mathrm{SeO}_{3}\right)_{2}, \quad \mathrm{~K}_{3} \mathrm{H}\left(\mathrm{CuP}_{2} \mathrm{O}_{7}\right)_{2}$, $\mathrm{Al}_{2} \mathrm{~F}_{2} \mathrm{CuSi}_{2} \mathrm{O}_{7}, \quad(\mathrm{La} / \mathrm{Gd} / \mathrm{Bi})_{2} \mathrm{Cu}\left(\mathrm{SeO}_{3}\right)_{4}, \quad \mathrm{Na}_{2} \mathrm{CuP}_{2} \mathrm{O}_{7}$, $(\mathrm{Sr} / \mathrm{Pb})_{2} \mathrm{Cu}\left(\mathrm{BO}_{3}\right)_{2}, \mathrm{Na}_{3} \mathrm{ClHCuPO}_{5}$, and $\mathrm{Ca}_{2} \mathrm{SbFeO}_{6}$. The second is the strong evidence for strong correlation physics, including antiferromagnetism and Mott insulating behavior, in the identified materials, based on density-functional theory (DFT) calculations incorporating electronic correlation corrections performed for a representative subset of five of the materials. Additionally, our findings illustrate the power of high-throughput computing and materials informatics to search for complex materials possessing rare electronic properties.

\section{METHODOLOGY}

\section{A. Screening strategy}

Our materials informatics screening approach consists of several materials properties that must be simultaneously satisfied.

(1) Chemistry. Compound must contain transition-metal and anion elements, and (for practicality) no radioactive elements.

(2) Crystal structure. In the compound, transition metals must be in a coordination whose local symmetry yields one or more singly degenerate $d$ orbitals based on crystal field theory. The coordinations considered are linear, trigonal planar, square planar, trigonal bipyramidal, square pyramidal, trigonal prismatic, pentagonal bipyramidal, and square antiprismatic.

(3) Electron count. All TMs in the compound must have a nominal $d$ electron count corresponding to a halffilled singly degenerate level given the crystal field splitting of the coordination environment.

(4) Thermodynamics. The compound must be no more than $25 \mathrm{meV} /$ atom above the thermodynamic ground state (as determined via convex hull analysis) and/or reported experimentally. This criterion is designed to focus on compounds which are likely to be synthesizable. The particular threshold value of $25 \mathrm{meV} /$ atom is chosen to match the magnitude of computed hull distances for synthesized metastable compounds found by Sun et al. for most chemistries [22].

Our search strategy is executed on the OQMD [23,24], an open database containing calculations of over half a million 
known and hypothetical inorganic crystalline compounds derived from the Inorganic Crystal Structure Database (ICSD) $[25,26]$ and structural prototypes. The OQMD contains electronic structure calculations at the DFT $[27,28]$ and in some cases DFT $+U$ level at consistent sets of parameters to enable consistent thermodynamic analysis.

We include details on the coordination environments considered in this study in the Supplemental Material [29]. A description of our methods for nominal electron counting and for the local structural queries performed to ascertain coordination environments are included in our previous work [30]. The coordination environment query relies on separate computation (outside of the QMPY framework [31]) of the TM nearest-neighbor bond lengths and angles, which are not directly stored in the OQMD.

Our screening strategy only requires computation of the electronic band structure as a postprocessing step for a small number of candidate materials. An alternative approach of directly analyzing the electronic band structures that exist in materials databases [32,33] is not applicable for our purpose primarily because such databases contain only band structures already including spin polarization and Hubbard $U$ effects, rather than the underlying non-spin-polarized DFT band structure. This is problematic since the desired isolated band, if present, can be hidden once it is spin split and pushed into other bands, as in the case of trigonal prismatic $\mathrm{VS}_{2}$ [20].

\section{B. Calculation details}

For the most promising materials identified, we perform additional DFT and DFT $+U$ calculations to investigate the electronic and magnetic properties in more detail. We employ the Vienna ab initio simulation package (VASP) [34-37] to perform generalized gradient approximation [38] calculations and employ the rotationally invariant Hubbard $U$ interaction [39]. We use the projector augmented wave method [40,41], a $600-\mathrm{eV}$ kinetic energy cutoff, and uniform $k$-point meshes of $k$-point density of $350 / \AA^{-3}$ or greater. The ionic forces and total energy are converged to $0.001 \mathrm{eV} / \AA$ and $10^{-6} \mathrm{eV}$, respectively. The high-symmetry $k$-point paths for band structures are based on the AFLOW conventions [42].

\section{RESULTS AND DISCUSSION}

\section{A. High-throughput materials screening}

We begin by discussing the number of materials obtained via our high-throughput materials screening strategy. Figure 1 illustrates how the number of candidate compounds is reduced from the total as successive filtering criteria are applied. Note that a logarithmic scale is used since many orders of magnitude are spanned. Removing compounds containing radioactive elements eliminates 120000 compounds from the total of 550000 candidates. We next filter by stability by including only compounds

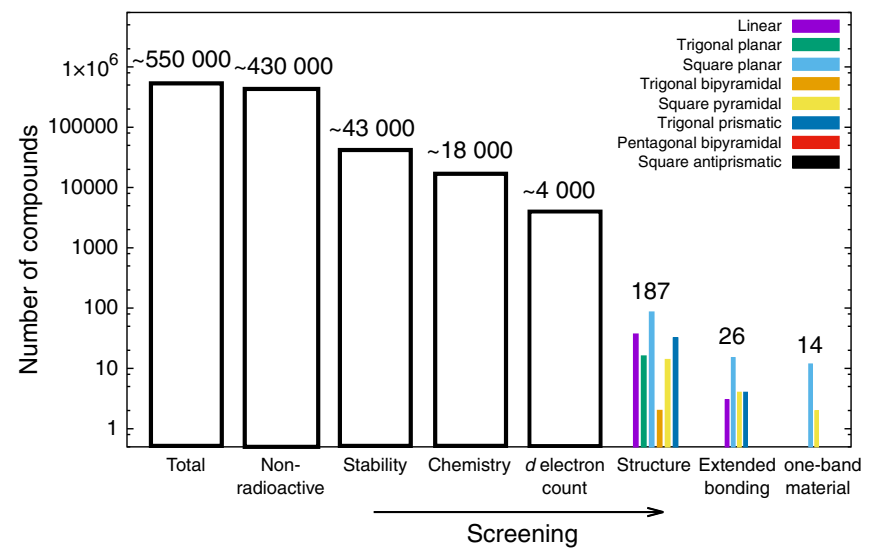

FIG. 1. Materials database screening for correlated one-band materials. The decrease in the number of compounds, on a logarithmic scale, is shown as additional screening criteria are applied from left to right.

which are thermodynamically stable or nearly stable (no more than $25 \mathrm{meV} /$ atom above the ground state). Irrespective of the computed stability, we also keep any compound if it has been reported experimentally. 43000 compounds remain after this filter. 18000 of the 43000 contain a TM and an anion and 4000 of these have a $d$ electron count compatible with one or more of the desired local coordination environments. Finally, we find that 187 of the compounds contain the corresponding coordination environment.

The two biggest decreases (in a fractional sense) of candidate compounds are (1) the stability filter, in which the number of compounds is reduced from 430000 to 43000 , and (2) the local structure filter, in which the number is reduced from 4000 to just 187 . The substantial decrease in candidate compounds from the stability filter, a general observation not tied specifically to searching for one-band correlated materials, is reflective of the high percentage of unstable, hypothetical compounds in the OQMD derived from structural prototypes. The significant decrease in candidates from the structural screening criterion reflects the infrequency of the low-degeneracy coordination environments that lead to singly degenerate $d$ orbital levels.

\section{B. Distribution of structure, chemistry, and thermodynamic stability}

We now describe the 187 candidate compounds identified by our screening strategy. The distribution of local TM coordinations for the 187 candidate compounds are displayed in Fig. 2(a). Square planar coordination is the most common, with nearly $50 \%$ of the total. Trigonal prismatic and linear coordinations are also found in significant numbers. Some of the more obscure coordinations like pentagonal bipyramidal are not found in a single compound.

Figure 2(b) illustrates the split of thermodynamic stabilities. Slightly more than half of the compounds found are 
(a)

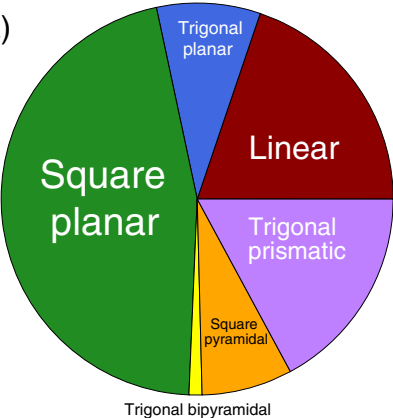

(b)

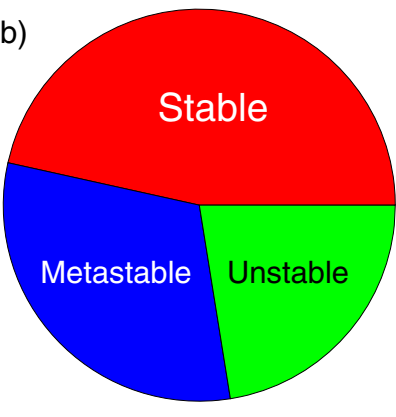

(c)

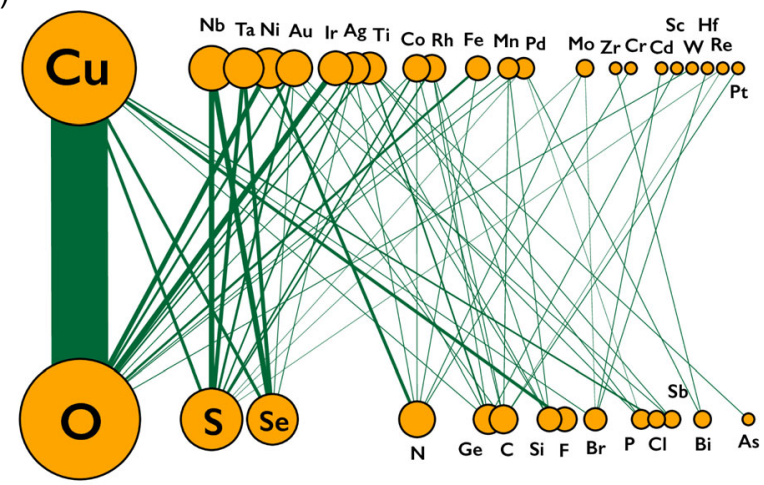

FIG. 2. Characterization of the identified compounds. Distribution of the 187 candidate compounds in terms of (a) TM coordination geometry, (b) thermodynamic stability, and (c) identity of the TM and its nearest-neighbor element.

thermodynamically stable, and around a third are nearly stable (no more than $25 \mathrm{meV} /$ atom above the ground state). The remaining compounds are more than $25 \mathrm{meV} /$ atom above the convex hull.

All but six of the 187 compounds identified originate from the ICSD. 104 of the 181 ICSD-derived compounds have one of 56 prototypes listed in the ICSD, which are shown in the Supplemental Material [29]. The most prevalent ICSD prototypes are trigonal planar $\mathrm{La}_{3} \mathrm{CuSiS}_{7}$-type (seven, e.g., $\mathrm{Sm}_{3} \mathrm{CuSnSe}_{7}$ ), trigonal prismatic $\mathrm{NbS}_{2}$-type (five, e.g., $\mathrm{TaSe}_{2}$ ), linear $\mathrm{CaTiO}_{3}$-type (five, e.g., $\mathrm{InCo}_{3} \mathrm{~N}$ ), and square planar $\mathrm{Ca}_{2} \mathrm{CuCl}_{2} \mathrm{O}_{2}$-type (five, e.g., $\mathrm{Sr}_{2} \mathrm{CoBr}_{2} \mathrm{O}_{2}$ ).

The only six hypothetical compounds derived from structural prototypes rather than the ICSD are $\mathrm{CsWO}_{3}$, $\mathrm{HfBr}_{3}, \mathrm{Mn}_{2} \mathrm{TeO}, \mathrm{BiPd}_{3}, \mathrm{Ni}_{3} \mathrm{Sb}$, and $\mathrm{Zr}_{3} \mathrm{Bi}$. The small number of such compounds suggests that a small fraction of the structural prototypes used to generate hypothetical compounds in the OQMD are well suited to achieve a oneband correlated material. For example, $L 1_{2}$ is currently the only OQMD prototype structure for which all sites of an element are in square planar coordination (e.g., the $\mathrm{Cu}$ site in $\left.\mathrm{CuAu}_{3}\right) . \mathrm{BiPd}_{3}, \mathrm{Ni}_{3} \mathrm{Sb}$, and $\mathrm{Zr}_{3} \mathrm{Bi}$ correspond to this prototype. $\mathrm{Mn}_{2} \mathrm{TeO}$ initially in the $\mathrm{Pnma}$ CaFeSeO structure [43] relaxes to a distinct Pnma structure with linear Mn-O coordination. $\mathrm{HfBr}_{3}$ initially in the $D 0_{19}\left(P 6_{3} / m m c, \mathrm{Ni}_{3} \mathrm{Sn}\right.$ type) structure relaxes to a distinct $P 6_{3} / \mathrm{mmc}$ structure with one-dimensional chains of triangular-face-sharing trigonal prismatic $\mathrm{HfBr}_{6}$ units. $\mathrm{CsWO}_{3}$ initially in the $R \overline{3}$ ilmenite $\left(\mathrm{FeTiO}_{3}\right.$-type) structure relaxes to a distinct $R \overline{3}$ structure with layers of edge-sharing trigonal prismatic $\mathrm{WO}_{6}$ units arranged as a kagome lattice.

The frequency of the TM and anion elements bonded in the low-degeneracy coordinations is illustrated as a network in Fig. 2(c). Here, each node represents a TM or anion element and the area of the node (width of the edge) is proportional to the number of compounds containing these element(s) in the low-degeneracy coordination. Copperoxygen is the most dominant chemistry with 74 of the 187 candidate compounds. $\mathrm{Cu}$ and $\mathrm{O}$ individually are the most prevalent TM and anion, respectively, as well: $\mathrm{Cu}$ is the TM in 90 of the compounds and $\mathrm{O}$ is the anion in 101 of the compounds. However, there are still many different TMs and anions represented. The anions $\mathrm{S}, \mathrm{Se}, \mathrm{N}$ and $\mathrm{TMs} \mathrm{Nb}$, $\mathrm{Ta}, \mathrm{Ni}$, and $\mathrm{Au}$ each occur in at least nine compounds. The presence of many $\mathrm{Cu}$-containing and square planar compounds is consistent with a high occurrence of square planar $\mathrm{Cu}^{2+}$ found in a recent statistical analysis of coordination environments [44].

The complete list of the 187 candidate compounds is included in the Supplemental Material [29]. We note that these 187 compounds include several well-known correlated materials such as the cuprate parent compound $\mathrm{La}_{2} \mathrm{CuO}_{4}$ and group- $\mathrm{V}$ transition metal dichalcogenides [e.g., $\left.(\mathrm{Nb} / \mathrm{Ta})(\mathrm{S} / \mathrm{Se})_{2}\right]$. More complicated materials such as the cuprate parent compound $\mathrm{YBa}_{2} \mathrm{Cu}_{3} \mathrm{O}_{7}$, which contains multiple $\mathrm{Cu}$ sites with distinct coordination environments and oxidation states, are not included. These results are suggestive of the ability of our screening strategy to find many, though not necessarily all, one-band correlated material candidates contained within our database.

\section{Additional screening criteria for non-cuprates with extended structures}

As discussed above, a key characteristic of strongly correlated materials like the cuprates is a large $U / t$ ratio. In this regime, the Coulomb repulsion can overwhelm the electron hopping, leading to localized electronic states and Mott insulating behavior as is found in, for example, the cuprate parent compounds. In addition to a large $U / t$ ratio, a finite hopping $t$ is still necessary to ensure there is a conduction pathway. As such, we expect materials with extended crystal structures (leading to extended hopping pathways and appreciable $t$ ) to be necessary to achieve a one-band correlated material.

In order to search for the most promising compounds among the 187 candidates for a one-band correlated material, we therefore perform an additional postprocessing screening criterion based on the crystal structures to discard any compound for which there is no connectivity (direct or indirect) between the TM-anion coordination cages. For example, $\mathrm{K}_{4} \mathrm{IrO}_{4}$ is removed since the $\mathrm{IrO}_{4}$ square planar units are isolated, whereas $\mathrm{TlCuPO}_{4}$ is retained since the 
TABLE I. The identified 26 noncuprate candidate compounds with extended electron hopping pathways and the TM coordination environment. The third column indicates whether the underlying electronic structure is found to be one-band in nature.

\begin{tabular}{|c|c|c|c|}
\hline \multicolumn{2}{|c|}{ Composition } & \multirow{2}{*}{$\frac{\text { Coordination }}{\text { Trig. prismatic }}$} & \multirow{2}{*}{$\frac{\text { One-band? }}{\ldots}$} \\
\hline Cs & $\mathrm{WO}_{3}$ & & \\
\hline $\mathrm{Li}$ & $\mathrm{MoN}_{2}$ & Trig. prismatic & $\ldots$ \\
\hline & $\mathrm{HfBr}_{3}$ & Trig. prismatic & $\cdots$ \\
\hline $\mathrm{Rb}$ & $\mathrm{Fe}\left(\mathrm{SeO}_{4}\right)_{2}$ & Trig. prismatic & $\cdots$ \\
\hline $\mathrm{K}_{3} \mathrm{H}$ & $\left(\mathrm{CuP}_{2} \mathrm{O}_{7}\right)_{2}$ & Sq. pyramidal & Yes \\
\hline $\mathrm{Al}_{2} \mathrm{~F}_{2}$ & $\mathrm{CuSi}_{2} \mathrm{O}_{7}$ & Sq. pyramidal & Yes \\
\hline $\mathrm{Ba}_{4}(\mathrm{Nd} / \mathrm{Sm})_{2}$ & $\mathrm{Cu}_{2} \mathrm{O}_{9}$ & Sq. pyramidal & $\cdots$ \\
\hline $\mathrm{Ca}$ & $\mathrm{NiN}$ & Linear & $\ldots$ \\
\hline $\mathrm{Sn}_{2}$ & $\mathrm{Co}_{3} \mathrm{~S}_{2}$ & Linear & $\cdots$ \\
\hline In & $\mathrm{Co}_{3} \mathrm{~N}$ & Linear & $\cdots$ \\
\hline$(\mathrm{Sr} / \mathrm{Ba})$ & $\mathrm{Cu}\left(\mathrm{SeO}_{3}\right)_{2}$ & Sq. planar & Yes \\
\hline & $\mathrm{CuBr}_{2}$ & Sq. planar & Yes \\
\hline$(\mathrm{La} / \mathrm{Gd} / \mathrm{Bi})_{2}$ & $\mathrm{Cu}\left(\mathrm{SeO}_{3}\right)_{4}$ & Sq. planar & Yes \\
\hline $\mathrm{Na}_{2}$ & $\mathrm{CuP}_{2} \mathrm{O}_{7}$ & Sq. planar & Yes \\
\hline $\mathrm{Tl}$ & $\mathrm{Cu}(\mathrm{P} / \mathrm{As}) \mathrm{O}_{4}$ & Sq. planar & $\ldots$ \\
\hline$(\mathrm{Sr} / \mathrm{Pb})_{2}$ & $\mathrm{Cu}\left(\mathrm{BO}_{3}\right)_{2}$ & Sq. planar & Yes \\
\hline $\mathrm{Ca}_{2} \mathrm{Sb}$ & $\mathrm{FeO}_{6}$ & Sq. planar & Yes \\
\hline $\mathrm{Ca}\left(\mathrm{H}_{2} \mathrm{O}\right)$ & $\mathrm{CuSiO}_{4}$ & Sq. planar & $\ldots$ \\
\hline $\mathrm{Li}_{2}$ & $\mathrm{CuO}_{2}$ & Sq. planar & Yes \\
\hline $\mathrm{Na}_{3} \mathrm{ClH}$ & $\mathrm{CuPO}_{5}$ & Sq. planar & Yes \\
\hline
\end{tabular}

$\mathrm{CuO}_{4}$ square planar units are connected indirectly via phosphate groups.

Finally, we also remove materials containing any of the $\mathrm{CuO}_{2}$ planes characteristic of the cuprates since this material class is already well studied and is not our focus. These two postprocessing steps reduce the number of candidate materials from 187 to 26; these candidates are contained in Table I and also shown in the "Extended bonding" screening criterion in Fig. 1. Only trigonal prismatic, square pyramidal, linear, and square planar coordinations remain after this additional screening step and compounds with $\mathrm{Cu}$ bonded to $\mathrm{O}$ continue to dominate.

\section{Electronic band structures}

For each of the 26 compounds, we compute the DFT electronic band structure to assess whether the underlying one-electron electronic structure is one-band in nature. The non-spin-polarized band structure is computed; possible effects of magnetism and strong electronic correlations on the electronic spectra are discussed in the following section. For simple crystal structures containing only a single TM atom in the primitive unit cell, the one-band condition corresponds to the presence of a single half-filled $d$ band straddling the Fermi energy with separations in energy below and above the band. For more complicated crystal structures with multiple TM atoms, we instead look for a single half-filled manifold of $d$ bands (containing no band gap) straddling the Fermi energy with separations in energy below and above the manifold, corresponding to one band per TM atom. We perform this assessment since, despite containing more than the literal "single" band, the band structures of such compounds should result in exactly the same one-orbital Hubbard physics as the first case. By requiring no overlap between the single band (or manifold of bands) and the remaining electronic bands, we are employing a stringent definition of a one-band material. Indeed, in the case of the cuprate parent compound $\mathrm{La}_{2} \mathrm{CuO}_{4}$, the single $d_{x^{2}-y^{2}}$ band does have some overlap with other bands [45]. However, our stringent definition (1) promotes the achievement of the single-orbital character and (2) is justified by our interest in finding a simple model system for the one-band Hubbard model.

The various screening criteria are not sufficient to ensure a one-band electronic structure, and many of the 26 candidates do not achieve the desired band structure. Several possible characteristics of the candidate compound can prevent the targeted band structure.

(1) Covalency. If there is too much hybridization between the TM $d$ states and anion $p$ states, an itinerant metal is found (e.g., $\mathrm{LiMoN}_{2}$ ).

(2) Metal-metal bonding. If the crystal structure has multiple TM sites and less localized $d$ states (e.g., $5 d$ ), these states can hybridize and form a band insulator by completely filling a bonding orbital (e.g., $\mathrm{CsWO}_{3}$ ).

(3) Avoided crossing. If the crystal structure has multiple TM sites, one can find a small-gap semiconductor due to an avoided crossing (e.g., $\mathrm{TlCuPO}_{4}$ ).

These false positive cases are discussed in the Supplemental Material [29].

We find 14 materials that successfully achieve a correlated one-band electronic structure: $\mathrm{Li}_{2} \mathrm{CuO}_{2}, \mathrm{CuBr}_{2}$ [46], $(\mathrm{Sr} / \mathrm{Ba}) \mathrm{Cu}\left(\mathrm{SeO}_{3}\right)_{2}, \quad \mathrm{~K}_{3} \mathrm{H}\left(\mathrm{CuP}_{2} \mathrm{O}_{7}\right)_{2}, \quad \mathrm{Al}_{2} \mathrm{~F}_{2} \mathrm{CuSi}_{2} \mathrm{O}_{7}$, $(\mathrm{La} / \mathrm{Gd} / \mathrm{Bi})_{2} \mathrm{Cu}\left(\mathrm{SeO}_{3}\right)_{4}, \mathrm{Na}_{2} \mathrm{CuP}_{2} \mathrm{O}_{7},(\mathrm{Sr} / \mathrm{Pb})_{2} \mathrm{Cu}\left(\mathrm{BO}_{3}\right)_{2}$, $\mathrm{Na}_{3} \mathrm{ClHCuPO}_{5}$, and $\mathrm{Ca}_{2} \mathrm{SbFeO}_{6}$, as indicated in Table I. These compounds correspond to the final "one-band material" label in Fig. 1. Figure 3 depicts the crystal structures and non-spin-polarized band structures of several of the 14 compounds: $\mathrm{CuBr}_{2}, \mathrm{Li}_{2} \mathrm{CuO}_{2}$, the selenates $\mathrm{BaCu}\left(\mathrm{SeO}_{3}\right)_{2}$ and $\mathrm{SrCu}\left(\mathrm{SeO}_{3}\right)_{2}$, and the pyrophosphate $\mathrm{K}_{3} \mathrm{H}\left(\mathrm{CuP}_{2} \mathrm{O}_{7}\right)_{2}$. We show these five compounds since they most clearly illustrate the underlying one-band electronic structure; the band structures of the other nine compounds are included in the Supplemental Material [29]. In the case of $\mathrm{CuBr}_{2}$ and $\mathrm{Li}_{2} \mathrm{CuO}_{2}$, there is a single $\mathrm{TM}$ atom in the primitive unit cell and a literal single band is found. While the selenates and pyrophosphate contain multiple TM atoms, the differences in TM atom environments are very small, such that the multiple bands straddling the Fermi energy exhibit only minor differences in dispersion. For example, along the high-symmetry $k$ path for $\mathrm{SrCu}\left(\mathrm{SeO}_{3}\right)_{2}$, the maximum splitting (at $\Gamma$ ) is only $34 \mathrm{meV}$. 

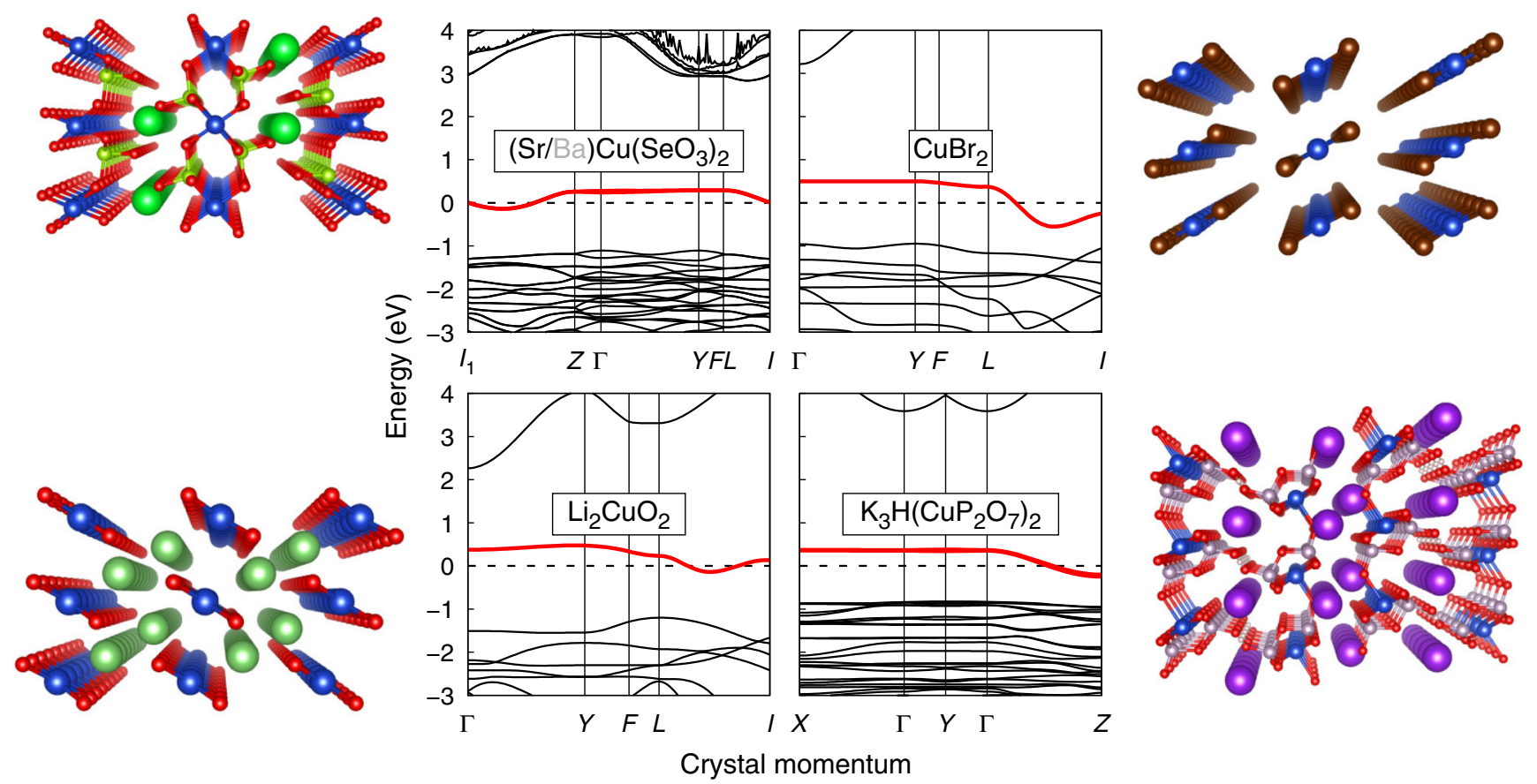

FIG. 3. Crystal structure and DFT electronic band structure of five of the identified one-band correlated materials. The single isolated half-filled electronic bands are shown as the thick red lines. The band structure of $\mathrm{BaCu}\left(\mathrm{SeO}_{3}\right)_{2}$ is not shown since it appears nearly identical to that of $\mathrm{SrCu}\left(\mathrm{SeO}_{3}\right)_{2}$.

All but one of the 14 identified compounds contain $\mathrm{Cu}$, suggesting it is difficult to achieve a one-band correlated material with other TMs. We find that the fraction of $\mathrm{Cu}$-based candidate materials in Fig. 1 increases gradually with successive filters, but the most significant increases occur for the last three (coordination, noncuprate with extended bonding, and one-band electronic structure). Despite the dominance of oxygen-containing compounds, we do find one compound lacking oxygen $\left(\mathrm{CuBr}_{2}\right)$. We also note similarity in the local structure of the compounds: all but $\mathrm{K}_{3} \mathrm{H}\left(\mathrm{CuP}_{2} \mathrm{O}_{7}\right)_{2}$ and $\mathrm{Al}_{2} \mathrm{~F}_{2} \mathrm{CuSi}_{2} \mathrm{O}_{7}$ have square planar coordination and these two are square pyramidal, which is closely related to square planar.

Other than three exceptions, the identified compounds are all stable or mildly metastable, i.e., within $30 \mathrm{meV} /$ atom of the convex hull (ground state). $\mathrm{Li}_{2} \mathrm{CuO}_{2}$ is $46 \mathrm{meV} /$ atom above the hull, $\mathrm{Na}_{3} \mathrm{ClHCuPO}_{5}$ is $143 \mathrm{meV} /$ atom above the hull, and $\mathrm{Ca}_{2} \mathrm{SbFeO}_{6}$ is $220 \mathrm{meV} /$ atom above the hull, but all three have been experimentally synthesized. Therefore, all 14 of the identified compounds should be ripe for synthesis and further experimental studies.

While $\mathrm{CuBr}_{2}$ is a binary compound with a simple stoichiometry, we also find compounds with much more complicated stoichiometries and structures. While $\mathrm{CuBr}_{2}$ and $\mathrm{Li}_{2} \mathrm{CuO}_{2}$ have $1 \mathrm{D}$ chains of edge-sharing $\mathrm{Cu}-\mathrm{O}$ square planar units, complex quasi-1D arrangement of the $\mathrm{Cu}-\mathrm{O}$ units are exhibited by $\mathrm{Na}_{2} \mathrm{CuP}_{2} \mathrm{O}_{7}, \mathrm{Na}_{3} \mathrm{ClHCuPO}_{5}$, and the borates. The remaining compounds have complicated $2 \mathrm{D}$ and $3 \mathrm{D}$ structures. $\mathrm{Na}_{3} \mathrm{ClHCuPO}_{5}$ is senary (six component elements) and even contains hydrogen. Our query just as easily finds these more complicated compounds, which is a strength of the informatics-driven approach to materials discovery.

\section{E. Evidence for strong correlation physics}

Finally, we discuss the possibility of strong correlation physics in the set of identified materials. In order to do so, we take the subset of materials mentioned above $\left[\mathrm{CuBr}_{7}\right.$, $\mathrm{Li}_{2} \mathrm{CuO}_{2}$, the selenates $(\mathrm{Ba} / \mathrm{Sr}) \mathrm{Cu}\left(\mathrm{SeO}_{3}\right)_{2}$, and the pyrophosphate $\mathrm{K}_{3} \mathrm{H}\left(\mathrm{CuP}_{2} \mathrm{O}_{7}\right)_{2}$ ] as representative examples and study them in more detail.

We note that even at the non-spin-polarized DFT level of theory, each of these five materials already shows potential for strong correlation physics. In particular, as shown in Fig. 3, each has a narrow (bandwidth no more than $1.1 \mathrm{eV}$ ) but not completely flat (bandwidth no less than $0.5 \mathrm{eV}$ ) half-filled band, which is suggestive of a large $U / t$ ratio and finite $t$, of substantial $d$ orbital character. Because of the relatively large size of the primitive unit cells for $(\mathrm{Sr} / \mathrm{Ba}) \mathrm{Cu}\left(\mathrm{SeO}_{3}\right)_{2}$ and $\mathrm{K}_{3} \mathrm{H}\left(\mathrm{CuP}_{2} \mathrm{O}_{7}\right)_{2}$, a quantitative calculation of the $U$ parameter is outside the scope of this work. However, previous estimations of $U$ for copper compounds based on ab initio calculations and experiments suggest values will very likely be on the order of $4 \mathrm{eV}$ or greater [39,47-62].

The non-spin-polarized band structure in Fig. 3 can be considered the underlying one-electron (band theory) electronic structure. Strong electron correlation can substantially modify the electronic structure in ways often not 


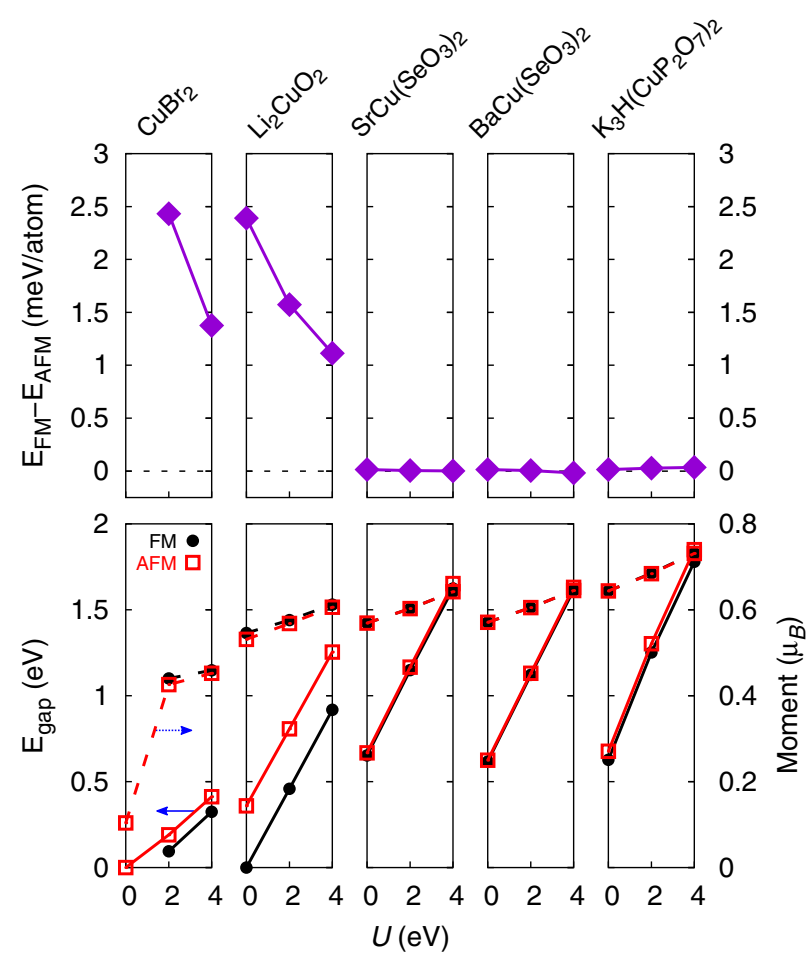

FIG. 4. Impact of electronic correlations via DFT $+U$ on the electronic and magnetic properties of the identified materials. The relative energetics of ferromagnetic (FM) and antiferromagnetic (AFM) states, electronic band gap, and local $\mathrm{Cu}$ magnetic moment as a function of correlation strength $U$ are shown for the subset of five candidate materials.

adequately captured by DFT, such as magnetism and Mott insulating behavior. Here, we go beyond non-spinpolarized DFT and use more sophisticated calculations incorporating magnetism and explicit on-site Coulomb interaction. The goal of such calculations (which still contain significant approximations) is not to perfectly describe the electronic properties of the identified materials, but rather to determine the possibility of strong correlation physics.

In particular, we perform spin-polarized DFT $+U$ calculations on the five candidate materials. Although DFT $+U$ corresponds to a mean-field solution to the local correlation problem (exactly solved via dynamical mean-field theory) [63], it gives a baseline expectation of the overall strength of the electronic correlations at a very cheap computational cost. The results of the DFT $(U=0)$ and DFT $+U$ (finite $U$ ) calculations including spin polarization are summarized in Fig. 4, which shows the dependence of the energetics, band gap, and magnetic moment on the on-site Coulomb repulsion $U$ for the five candidate materials. Several aspects suggest interesting strong correlation behavior. At the spin-polarized DFT level, all but $\mathrm{CuBr}_{2}$ exhibit a magnetic instability corresponding to the formation of local moments. All but $\mathrm{CuBr}_{2}$ not only become magnetic, but they all fully spin polarize and open up a band gap (forming a $S=1 / 2$ state).
DFT $+U$ calculations for $U=2$ and $4 \mathrm{eV}$ show that for all of these materials (even the bromide), all of the different magnetic configurations (ferromagnetic and antiferromagnetic) are gapped. This suggests Mott insulating behavior in which the strong Coulomb interaction, rather than a particular magnetic configuration, leads to an insulating state. In $\mathrm{CuBr}_{2}$ and $\mathrm{Li}_{2} \mathrm{CuO}_{2}$, antiferromagnetism is preferred over ferromagnetism as in the cuprates. In contrast, in the more complicated crystal structures (the selenates and pyrophosphate), we find that the ferromagnetic and antiferromagnetic states are essentially degenerate (less than $1 \mathrm{meV} /$ atom energy difference). This suggests that in these materials there is a very weak magnetic coupling, as might be expected since the square planar and square pyramidal units are not in close proximity. Since the magnetic coupling goes as $t^{2} / U$, the weaker magnetic coupling is consistent with the smaller bandwidth for these compounds as compared to those of $\mathrm{CuBr}_{2}$ and $\mathrm{Li}_{2} \mathrm{CuO}_{2}$.

The identified materials, whose underlying singleelectron band structure is one-band in nature, exhibit a strong tendency for the formation of insulating states with fully polarized magnetic moments regardless of magnetic configuration, as well as the presence of antiferromagnetic ordering in some cases. While more accurate correlated calculations and experiments will be necessary to elucidate the true electronic structure, these results at the approximate DFT $+U$ level already represent strong evidence for the possibility of interesting strong correlation physics in these materials candidates for the rare one-band correlated material.

\section{CONCLUSIONS}

We employ a materials informatics approach to search for one-band correlated materials analogous to the cuprate high-temperature superconductors. Using a query based on transition-metal $d$ electron count, crystal field theory, and formation energy, we search the more than a half million real and hypothetical inorganic crystals in the Open Quantum Materials Database for synthesizable materials with an isolated half-filled $d$ band in the low-energy spectrum. Density-functional theory band structure calculations illustrate that $13 \mathrm{Cu}$ compounds, including bromide, oxide, selenate, borate, pyrophosphate, hydrogen phosphate, and pyrosilicate chemistries, and one Fe oxide compound, successfully achieve the one-band electronic structure. Significant evidence of strong correlation physics in the candidate compounds, including Mott insulating behavior and antiferromagnetism, is revealed by further calculations including magnetism and on-site Coulomb interactions performed on a representative subset of five of the materials. Our data-driven approach opens up new possibilities for the design and discovery of materials with rare electronic properties. 


\section{ACKNOWLEDGMENTS}

We acknowledge support from the U.S. Department of Energy under Contract No. DE-SC0015106. Computational resources were provided by the Quest high performance computing facility at Northwestern University, the National Energy Research Scientific Computing Center (U.S. Department of Energy Contract No. DE-AC0205CH11231), and the Extreme Science and Engineering Discovery Environment (National Science Foundation Contract No. ACI-1548562).

[1] J. G. Bednorz and K. A. Müller, Possible High $T_{c}$ Superconductivity in the Ba-La-Cu-O System, Z. Phys. B 64, 189 (1986).

[2] P. A. Lee, N. Nagaosa, and X.-G. Wen, Doping a Mott Insulator: Physics of High-Temperature Superconductivity, Rev. Mod. Phys. 78, 17 (2006).

[3] W. E. Pickett, Electronic Structure of the High-Temperature Oxide Superconductors, Rev. Mod. Phys. 61, 433 (1989).

[4] E. H. Lieb and F. Y. Wu, Absence of Mott Transition in an Exact Solution of the Short-Range, One-Band Model in One Dimension, Phys. Rev. Lett. 20, 1445 (1968).

[5] A. Georges and G. Kotliar, Hubbard Model in Infinite Dimensions, Phys. Rev. B 45, 6479 (1992).

[6] B. J. Kim, H. Jin, S. J. Moon, J.-Y. Kim, B.-G. Park, C. S. Leem, J. Yu, T. W. Noh, C. Kim, S.-J. Oh, J.-H. Park, V. Durairaj, G. Cao, and E. Rotenberg, Novel $J_{\text {eff }}=1 / 2$ Mott State Induced by Relativistic Spin-Orbit Coupling in $\mathrm{Sr}_{2} \mathrm{IrO}_{4}$, Phys. Rev. Lett. 101, 076402 (2008).

[7] J. Chaloupka and G. Khaliullin, Orbital Order and Possible Superconductivity in $\mathrm{LaNiO}_{3} / \mathrm{LaMO}_{3}$ Superlattices, Phys. Rev. Lett. 100, 016404 (2008).

[8] M. Wu, E. Benckiser, M. W. Haverkort, A. Frano, Y. Lu, U. Nwankwo, S. Brück, P. Audehm, E. Goering, S. Macke, V. Hinkov, P. Wochner, G. Christiani, S. Heinze, G. Logvenov, H.-U. Habermeier, and B. Keimer, Strain and Composition Dependence of Orbital Polarization in Nickel Oxide Superlattices, Phys. Rev. B 88, 125124 (2013).

[9] S. M. Griffin, P. Staar, T. C. Schulthess, M. Troyer, and N. A. Spaldin, A Bespoke Single-Band Hubbard Model Material, Phys. Rev. B 93, 075115 (2016).

[10] S. Curtarolo, G. L. W. Hart, M. B. Nardelli, N. Mingo, S. Sanvito, and O. Levy, The High-Throughput Highway to Computational Materials Design, Nat. Mater. 12, 191 (2013).

[11] A. Agrawal and A. Choudhary, Perspective: Materials Informatics and Big Data: Realization of the Fourth Paradigm of Science in Materials Science, APL Mater. 4, 053208 (2016).

[12] A. Jain, G. Hautier, S. P. Ong, and K. Persson, New Opportunities for Materials Informatics: Resources and Data Mining Techniques for Uncovering Hidden Relationships, J. Mater. Res. 31, 977 (2016).

[13] H. Rosner, H. Eschrig, R. Hayn, S.-L. Drechsler, and J. Málek, Electronic Structure and Magnetic Properties of the Linear Chain Cuprates $\mathrm{Sr}_{2} \mathrm{CuO}_{3}$ and $\mathrm{Ca}_{2} \mathrm{CuO}_{3}$, Phys. Rev. B 56, 3402 (1997).
[14] H. Rosner, S.-L. Drechsler, K. Koepernik, R. Hayn, and H. Eschrig, Possibility of a Spin-Peierls State in $\mathrm{CuSiO}_{3}$ from Electronic Structure Theory, Phys. Rev. B 63, 073104 (2001).

[15] R. Nath, D. Kasinathan, H. Rosner, M. Baenitz, and C. Geibel, Electronic and Magnetic Properties of $\mathrm{K}_{2} \mathrm{CuP}_{2} \mathrm{O}_{7}$ : A Model $S=\frac{1}{2}$ Heisenberg Chain System, Phys. Rev. B 77, 134451 (2008).

[16] M. Schmitt, O. Janson, M. Schmidt, S. Hoffmann, W. Schnelle, S.-L. Drechsler, and H. Rosner, Crystal-WaterInduced Switching of Magnetically Active Orbitals in $\mathrm{CuCl}_{2}$, Phys. Rev. B 79, 245119 (2009).

[17] O. Janson, W. Schnelle, M. Schmidt, Yu Prots, S.-L. Drechsler, S. K. Filatov, and H. Rosner, Electronic Structure and Magnetic Properties of the Spin-1/2 Heisenberg System $\mathrm{CuSe}_{2} \mathrm{O}_{5}$, New J. Phys. 11, 113034 (2009).

[18] J. M. Rondinelli and N. A. Spaldin, Electron-Lattice Instabilities Suppress Cuprate-like Electronic Structures in $\mathrm{SrFeO}_{3} / \mathrm{SrTiO}_{3}$ Superlattices, Phys. Rev. B 81, 085109 (2010).

[19] M. Klintenberg and O. Eriksson, Possible HighTemperature Superconductors Predicted from Electronic Structure and Data-Filtering Algorithms, Comput. Mater. Sci. 67, 282 (2013).

[20] E. B. Isaacs and C. A. Marianetti, Electronic Correlations in Monolayer $\mathrm{VS}_{2}$, Phys. Rev. B 94, 035120 (2016).

[21] A. S. Botana, V. Pardo, and M. R. Norman, Electron Doped Layered Nickelates: Spanning the Phase Diagram of the Cuprates, Phys. Rev. Mater. 1, 021801 (2017).

[22] W. Sun, S. T. Dacek, S. P. Ong, G. Hautier, A. Jain, W. D. Richards, A. C. Gamst, K. A. Persson, and G. Ceder, The Thermodynamic Scale of Inorganic Crystalline Metastability, Sci. Adv. 2, e1600225 (2016).

[23] J.E. Saal, S. Kirklin, M. Aykol, B. Meredig, and C. Wolverton, Materials Design and Discovery with HighThroughput Density Functional Theory: The Open Quantum Materials Database (OQMD), JOM 65, 1501 (2013).

[24] S. Kirklin, J. E. Saal, B. Meredig, A. Thompson, J. W. Doak, M. Aykol, S. Rühl, and C. Wolverton, The Open Quantum Materials Database (OQMD): Assessing the Accuracy of DFT Formation Energies, npj Comput. Mater. 1, 15010 (2015).

[25] G. Bergerhoff, R. Hundt, R. Sievers, and I. D. Brown, The Inorganic Crystal Structure Data Base, J. Chem. Inf. Comput. Sci. 23, 66 (1983).

[26] A. Belsky, M. Hellenbrandt, V. L. Karen, and P. Luksch, New Developments in the Inorganic Crystal Structure Database (ICSD): Accessibility in Support of Materials Research and Design, Acta Crystallogr. Sect. B 58, 364 (2002).

[27] P. Hohenberg and W. Kohn, Inhomogeneous Electron Gas, Phys. Rev. 136, B864 (1964).

[28] W. Kohn and L. J. Sham, Self-Consistent Equations Including Exchange and Correlation Effects, Phys. Rev. 140, A1133 (1965).

[29] See Supplemental Material at http://link.aps.org/ supplemental/10.1103/PhysRevX.9.021042 for details on the coordination environment definitions, the band structures of the identified materials, discussion of false positive examples, and the complete list of 187 candidate compounds. 
[30] E. B. Isaacs and C. Wolverton, Inverse Band Structure Design via Materials Database Screening: Application to Square Planar Thermoelectrics, Chem. Mater. 30, 1540 (2018).

[31] QMPY, https://github.com/wolverton-research-group/qmpy.

[32] S. Curtarolo, W. Setyawan, S. Wang, J. Xue, K. Yang, R. H. Taylor, L. J. Nelson, G. L. W. Hart, S. Sanvito, M. Buongiorno-Nardelli, N. Mingo, and O. Levy, AFLOWLIB.ORG: A Distributed Materials Properties Repository from High-Throughput Ab Initio Calculations, Comput. Mater. Sci. 58, 227 (2012).

[33] A. Jain, S. P. Ong, G. Hautier, W. Chen, W. D. Richards, S. Dacek, S. Cholia, D. Gunter, D. Skinner, G. Ceder, and K. A. Persson, Commentary: The Materials Project: A Materials Genome Approach to Accelerating Materials Innovation, APL Mater. 1, 011002 (2013).

[34] G. Kresse and J. Hafner, Ab Initio Molecular-Dynamics Simulation of the Liquid-Metal-Amorphous-Semiconductor Transition in Germanium, Phys. Rev. B 49, 14251 (1994).

[35] G. Kresse and J. Hafner, Ab Initio Molecular Dynamics for Liquid Metals, Phys. Rev. B 47, 558 (1993).

[36] G. Kresse and J. Furthmüller, Efficient Iterative Schemes for Ab Initio Total-Energy Calculations Using a Plane-Wave Basis Set, Phys. Rev. B 54, 11169 (1996).

[37] G. Kresse and J. Furthmüller, Efficiency of Ab-Initio Total Energy Calculations for Metals and Semiconductors Using a Plane-Wave Basis Set, Comput. Mater. Sci. 6, 15 (1996).

[38] J. P. Perdew, K. Burke, and M. Ernzerhof, Generalized Gradient Approximation Made Simple, Phys. Rev. Lett. 77, 3865 (1996).

[39] A. I. Liechtenstein, V. I. Anisimov, and J. Zaanen, DensityFunctional Theory and Strong Interactions: Orbital Ordering in Mott-Hubbard Insulators, Phys. Rev. B 52, R5467 (1995).

[40] P. E. Blöchl, Projector Augmented-Wave Method, Phys. Rev. B 50, 17953 (1994).

[41] G. Kresse and D. Joubert, From Ultrasoft Pseudopotentials to the Projector Augmented-Wave Method, Phys. Rev. B 59, 1758 (1999).

[42] W. Setyawan and S. Curtarolo, High-Throughput Electronic Band Structure Calculations: Challenges and Tools, Comput. Mater. Sci. 49, 299 (2010).

[43] F. Han, D. Wang, C. D. Malliakas, M. Sturza, D. Y. Chung, X. Wan, and M. G. Kanatzidis, $(\mathrm{CaO})(\mathrm{FeSe})$ : A Layered Wide-Gap Oxychalcogenide Semiconductor, Chem. Mater. 27, 5695 (2015).

[44] D. Waroquiers, X. Gonze, G.-M. Rignanese, C. WelkerNieuwoudt, F. Rosowski, M. Göbel, S. Schenk, P. Degelmann, R. André, R. Glaum, and G. Hautier, Statistical Analysis of Coordination Environments in Oxides, Chem. Mater. 29, 8346 (2017).

[45] H. Sakakibara, H. Usui, K. Kuroki, R. Arita, and H. Aoki, Origin of the Material Dependence of $T_{c}$ in the Single-Layered Cuprates, Phys. Rev. B 85, 064501 (2012).

[46] C. Lee, J. Liu, M.-H. Whangbo, H.-J. Koo, R. K. Kremer, and A. Simon, Investigation of the Spin Exchange
Interactions and the Magnetic Structure of the High-Temperature Multiferroic $\mathrm{CuBr}_{2}$, Phys. Rev. B 86, 060407(R) (2012).

[47] V. I. Anisimov, J. Zaanen, and O. K. Andersen, Band Theory and Mott Insulators: Hubbard U Instead of Stoner I, Phys. Rev. B 44, 943 (1991).

[48] V. R. Galakhov, A. I. Poteryaev, E. Z. Kurmaev, V. I. Anisimov, S. Bartkowski, M. Neumann, Z. W. Lu, B. M. Klein, and T.-R. Zhao, Valence-Band Spectra and Electronic Structure of $\mathrm{CuFeO}_{2}$, Phys. Rev. B 56, 4584 (1997).

[49] V. I. Anisimov, M. A. Korotin, I. A. Nekrasov, Z. V. Pchelkina, and S. Sorella, First Principles Electronic Model for High-Temperature Superconductivity, Phys. Rev. B 66, 100502(R) (2002).

[50] V. I. Anisimov, M. A. Korotin, A. S. Mylnikova, A. V. Kozhevnikov, D. M. Korotin, and J. Lorenzana, Computation of Stripes in Cuprates within the LDA $+U$ Method, Phys. Rev. B 70, 172501 (2004).

[51] L. Wang, T. Maxisch, and G. Ceder, Oxidation Energies of Transition Metal Oxides within the GGA + U Framework, Phys. Rev. B 73, 195107 (2006).

[52] M. Nolan and S. D. Elliott, The p-Type Conduction Mechanism in $\mathrm{Cu}_{2} \mathrm{O}$ : A First Principles Study, Phys. Chem. Chem. Phys. 8, 5350 (2006).

[53] V. V. Mazurenko, S. L. Skornyakov, A. V. Kozhevnikov, F. Mila, and V. I. Anisimov, Wannier Functions and Exchange Integrals: The Example of $\mathrm{LiCu}_{2} \mathrm{O}_{2}$, Phys. Rev. B 75, 224408 (2007).

[54] A. Önsten, M. Månsson, T. Claesson, T. Muro, T. Matsushita, T. Nakamura, T. Kinoshita, U. O. Karlsson, and O. Tjernberg, Probing the Valence Band Structure of $\mathrm{Cu}_{2} \mathrm{O}$ Using HighEnergy Angle-Resolved Photoelectron Spectroscopy, Phys. Rev. B 76, 115127 (2007).

[55] A. Jain, G. Hautier, S. P. Ong, C. J. Moore, C. C. Fischer, K. A. Persson, and G. Ceder, Formation Enthalpies by Mixing GGA and GGA + U Calculations, Phys. Rev. B 84, 045115 (2011).

[56] B. Himmetoglu, R. M. Wentzcovitch, and M. Cococcioni, First-Principles Study of Electronic and Structural Properties of CuO, Phys. Rev. B 84, 115108 (2011).

[57] L. Y. Isseroff and E. A. Carter, Importance of Reference Hamiltonians Containing Exact Exchange for Accurate One-Shot GW Calculations of $\mathrm{Cu}_{2} \mathrm{O}$, Phys. Rev. B 85, 235142 (2012).

[58] C. E. Ekuma, V. I. Anisimov, J. Moreno, and M. Jarrell, Electronic Structure and Spectra of $\mathrm{CuO}$, Eur. Phys. J. B 87, 23 (2014).

[59] G. W. Mann, K. Lee, M. Cococcioni, B. Smit, and J. B. Neaton, First-Principles Hubbard U Approach for Small Molecule Binding in Metal-Organic Frameworks, J. Chem. Phys. 144, 174104 (2016).

[60] J. A. Suárez, J. J. Plata, A. M. Márquez, and J. F. Sanz, Structural, Electronic and Optical Properties of Copper, Silver and Gold Sulfide: A DFT Study, Theor. Chem. Acc. 135, 70 (2016).

[61] I. E. Brumboiu, S. Haldar, J. Lüder, O. Eriksson, H. C. Herper, B. Brena, and B. Sanyal, Influence of Electron 
Correlation on the Electronic Structure and Magnetism of Transition-Metal Phthalocyanines, J. Chem. Theory Comput. 12, 1772 (2016).

[62] M. J. Cliffe, J. Lee, J. A. M. Paddison, S. Schott, P. Mukherjee, M. W. Gaultois, P. Manuel, H. Sirringhaus, S. E. Dutton, and C. P. Grey, Low-Dimensional Quantum
Magnetism in $\mathrm{Cu}(\mathrm{NCS})_{2}:$ A Molecular Framework Material, Phys. Rev. B 97, 144421 (2018).

[63] G. Kotliar, S. Y. Savrasov, K. Haule, V. S. Oudovenko, O. Parcollet, and C. A. Marianetti, Electronic Structure Calculations with Dynamical Mean-Field Theory, Rev. Mod. Phys. 78, 865 (2006). 